\title{
CHARACTERIZATIONS OF CENTRAL SYMMETRY FOR CONVEX BODIES IN MINKOWSKI SPACES
}

\author{
GENNADIY AVERKOV ${ }^{1 *}$, ENDRE MAKAI, JR. ${ }^{2 * *}$ and HORST MARTINI ${ }^{1 * * *}$ \\ 1 Mathematical Faculty, University of Technology, D-09107 Chemnitz, Germany \\ e-mails: gennadiy.averkov@googlemail.com, martini@mathematik.tu-chemnitz.de \\ 2 A. Rényi Institute of Mathematics, Hungarian Academy of Sciences, Pf. 127, \\ H-1364 Budapest, Hungary \\ e-mail: makai@renyi.hu
}

Communicated by G. Fejes Tóth

(Received April 25, 2007; accepted November 5, 2007)

\begin{abstract}
K. Zindler [47] and P. C. Hammer and T. J. Smith [19] showed the following: Let $K$ be a convex body in the Euclidean plane such that any two boundary points $p$ and $q$ of $K$, that divide the circumference of $K$ into two arcs of equal length, are antipodal. Then $K$ is centrally symmetric. [19] announced the analogous result for any Minkowski plane $\mathbb{M}^{2}$, with arc length measured in the respective Minkowski metric. This was recently proved by Y. D. Chai-Y. I. Kim [7] and G. Averkov [4]. On the other hand, for Euclidean $d$-space $\mathbb{R}^{d}, \mathrm{R}$. Schneider [38] proved that if $K \subset \mathbb{R}^{d}$ is a convex body, such that each shadow boundary of $K$ with respect to parallel illumination halves the Euclidean surface area of $K$ (for the definition of "halving" see in the paper), then $K$ is centrally symmetric. (This implies the result from [19] for $\mathbb{R}^{2}$.) We give a common generalization of the results of Schneider [38] and Averkov [4]. Namely, let $\mathbb{M}^{d}$ be a $d$-dimensional Minkowski space, and $K \subset \mathbb{M}^{d}$ be a convex body. If some Minkowskian surface area (e.g., Busemann's or Holmes-Thompson's) of $K$ is halved by each shadow boundary of $K$ with respect to parallel illumination, then $K$ is centrally symmetric. Actually, we use little from the definition of Minkowskian surface area(s). We may measure "surface area" via any even Borel func-
\end{abstract}

2000 Mathematics Subject Classification. Primary 52A20; Secondary 52A21, 33C55.

Key words and phrases. Antipodality, Busemann definition of surface area, central symmetry, convex body, Holmes-Thompson definition of surface area, hemispherical transformation, illumination, Minkowski space, (Minkowskian) surface area measure, normed linear space, (sharp) shadow boundary, spherical harmonics.

${ }^{*}$ Research supported by Deutsche Forschungsgemeinschaft, Project AV 85/1-1.

** Research partially supported by Hungarian National Foundation for Scientific Research, Grant Nos. T046846, T043520.

*** The author was supported by the "Discrete and Convex" project (MTKD-CT-2005014333) carried out by Alfréd Rényi Institute of Mathematics - Hungarian Academy of Sciences, in the framework of the European Community's "Structuring the European Research Area" program. 
tion $\varphi: S^{d-1} \rightarrow \mathbb{R}$, for a convex body $K$ with Euclidean surface area measure $d S_{K}(u)$, with $\varphi(u)$ being $d S_{K}(u)$-almost everywhere non-0, by the formula $B \mapsto \int_{B} \varphi(u) d S_{K}(u)$ (supposing that $\varphi$ is integrable with respect to $d S_{K}(u)$ ), for $B \subset S^{d-1}$ a Borel set, rather than the Euclidean surface area measure $B \mapsto \int_{B} d S_{K}(u)$. The conclusion remains the same, even if we suppose surface area halving only for parallel illumination from almost all directions. Moreover, replacing the surface are a measure $d S_{K}(u)$ by the $k$-th area measure of $K$ ( $k$ with $1 \leqq k \leqq d-2$ an integer), the analogous result holds. We follow rather closely the proof for $\mathbb{R}^{d}$, which is due to Schneider [38].

\section{Preliminaries}

Let $\mathbb{R}^{d}$ denote the $d$-dimensional Euclidean space, with unit sphere $S^{d-1}$, where $d \geqq 2$. A convex body $K \subset \mathbb{R}^{d}$ is a compact, convex set with nonempty interior. The volume of $K$ will be denoted by $V(K)$. A real normed linear space of dimension $d$ is called a Minkowski space and denoted by $\mathbb{M}^{d}$ (i.e., $\mathbb{R}^{d}$, endowed with some Minkowski metric), whose unit ball is a convex body centred at the origin, and whose origin will be denoted by $O$. The geometry of Minkowski spaces is intensively studied in [43].

Definition 1.1. Let $K \subset \mathbb{R}^{d}$ be a convex body, and $p_{1}, p_{2}$ be two points of $K$. We say that $p_{1}, p_{2}$ are antipodal points of $K$, if there are different parallel supporting hyperplanes $H_{1}, H_{2}$ of $K$, such that $p_{1} \in H_{1}, p_{2} \in H_{2}$ (of course, then $p_{1}, p_{2} \in \mathrm{bd} K$, the boundary of $K$ ).

Properties of pairs of antipodal points of finite sets (i.e., of their convex hulls, but belonging to the finite sets) and of convex bodies are surveyed in $[31]$.

Definition 1.2. Let $K \subset \mathbb{R}^{d}$ be a convex body, and $u \in S^{d-1}$ be an arbitrary direction. The shadow boundary of $K$ with respect to parallel illumination from direction $u$ is the set $(\mathrm{bd} K) \cap \mathrm{bd}\left(\pi_{u}^{-1}\left(\pi_{u} K\right)\right)$, where $\pi_{u}$ is the orthogonal projection of $\mathbb{R}^{d}$ to the linear $(d-1)$-dimensional subspace orthogonal to $u$. Such a shadow boundary is sharp, if the restriction of $\pi_{u}$ to the shadow boundary in question establishes a bijection between the shadow boundary and rel bd $\left(\pi_{u} K\right)$, where the relative boundary rel bd is meant in the linear $(d-1)$-subspace orthogonal to $u$.

For various properties and applications of shadow boundaries of convex bodies we refer to [29], [28], and [11].

Definition 1.3. Let $\mathbb{M}^{2}$ be a Minkowski plane with norm $\|\cdot\|$. The Minkowski length $\|d s\|$ of an arc element ds is defined as $|d s| /|O P|$, where $|d s|$ is the Euclidean arc length of the arc element $d s$, and $d s$ and $O P$ are parallel and of the same orientation, $|O P|$ is the Euclidean length of $O P$, and the Minkowski length $\|O P\|$ of the segment $O P$ is 1 . The Minkowski length of an arc on the boundary of a convex body is defined as $\int\|d s\|$, the domain of integration being the arc under consideration. 
Contrary to the two-dimensional situation, there is no unique definition of Minkowskian surface area for $d \geqq 3$.

Let $d \geqq 2$, and $K \subset \mathbb{R}^{d}$ be a convex body. We write $d S_{K}(u)$, with $u \in S^{d-1}$, for the surface area measure of $K$. This is a non-negative finite measure defined on the Borel sets of $S^{d-1}$, and its value at a Borel set $B \subset S^{d-1}$ is the $(d-1)$-dimensional Hausdorff measure of $\{x \in \operatorname{bd} K \mid \exists u$ which is an outer normal unit vector of $K$ at $x$ with $u \in B\}$, see [40], Theorem 4.2.5. Note that $u \in S^{d-1}$ is an outer normal unit vector of $K$ at $x \in \operatorname{bd} K$ if $\langle x, u\rangle=\max \{\langle y, u\rangle \mid y \in K\}$.

In the monograph [43], p. 137, several requirements for the definition of Minkowskian surface area are listed. A Minkowskian surface area measure of a convex body $K \subset \mathbb{M}^{d}$ is a non-negative finite measure defined on the Borel sets of $S^{d-1}$, whose value at a Borel set $B \subset S^{d-1}$ is given as

$$
\int_{B} d S_{K}(u) / f\left(U \cap u^{\perp}\right),
$$

where $U$ is the unit ball of the Minkowski space $\mathbb{M}^{d}$, and $u^{\perp}$ is the linear $(d-1)$-dimensional subspace of $\mathbb{R}^{d}$ orthogonal to $u \in S^{d-1}\left(S^{d-1}\right.$, orthogonality and $d S_{K}(u)$ meant with respect to a fixed underlying Euclidean metric on $\left.\mathbb{M}^{d}\right)$. Further, $f$ is a positive continuous function defined for $(d-1)$ dimensional $O$-symmetric convex bodies $L \subset \mathbb{R}^{d}$, where $V_{d-1}(L) / f(L)$ is invariant under taking images of $L$ under non-singular linear transformations of $\mathbb{M}^{d}$, and $V_{d-1}$ denotes $(d-1)$-dimensional volume. (Thus the Euclidean $(d-1)$-dimensional measure in translates of $u^{\perp}$ normalized (i.e., divided) by the magnitude $f\left(U \cap u^{\perp}\right)$ is the Minkowskian surface area measure in translates of $u^{\perp}$.) In particular, $f\left(U \cap u^{\perp}\right)$ is an even, positive continuous function of $u \in S^{d-1}$. Still it is required that for Euclidean space $f\left(U \cap u^{\perp}\right)$ should be equal to $\kappa_{d-1}$, the Euclidean $(d-1)$-volume of the Euclidean unit $(d-1)$-ball should be equal to 1 , hence the Minkowskian surface area measure should be equal to the usual Euclidean surface area measure. Moreover, it is required that the set $\left\{f\left(U \cap u^{\perp}\right) u \mid u \in S^{d-1}\right\}$ be the boundary of some $\left(O\right.$-symmetric) convex body $M \subset \mathbb{R}^{d}$. Then the Minkowskian surface area measure is the set function, defined on Borel sets $B$ of $S^{d-1}$ by $\int_{B} h_{M^{*}}(u) d S_{K}(u)$, where $M^{*}$ is the polar reciprocal of $M$ with respect to the Euclidean unit ball centred at $O$, and $h_{M^{*}}(u)$ is the support function of $M^{*}$. (A suitable multiple of $M^{*}$ is usually called the isoperimetrix associated to the function $f$, cf. [43], p. 149.)

In Chapters 5, 6, and 7 of his monograph [43] A. C. Thompson deals with two Minkowskian surface area measures satisfying all postulates given above. That of Busemann is defined by $f(L)=V_{d-1}(L) / \kappa_{d-1}$, and that of 
Holmes-Thompson is defined by $f(L)=\kappa_{d-1}^{2} / V_{d-1}\left(L^{*}\right)$, where $L^{*}$ is the polar reciprocal of $L$ in its linear hull, with respect to the intersection of the Euclidean unit ball about $O$ and the linear hull of $L$. Here $\kappa_{d-1}$ is the Euclidean $(d-1)$-volume of the Euclidean unit $(d-1)$-ball.

DeFinition 1.4. Let $K$ be a convex body in a $d$-dimensional Minkowski space $\mathbb{M}^{d}$. Let $u \in S^{d-1}$ be a direction $\left(S^{d-1}\right.$ meant again with respect to a fixed underlying Euclidean metric on $\mathbb{M}^{d}$ ). We say that the shadow boundary with respect to parallel illumination from direction u halves the Minkowskian surface area of $K$, defined by some specific function $f$ satisfying the requirements prescribed above for these functions $f$, if for this $u \in S^{d-1}$ we have

$$
\int_{S_{u}^{+}} d S_{K}(u) / f\left(U \cap u^{\perp}\right)=\int_{S_{u}^{-}} d S_{K}(u) / f\left(U \cap u^{\perp}\right),
$$

where $S_{u}^{+}:=\left\{v \in S^{d-1} \mid\langle u, v\rangle \geqq 0\right\}$ and $S_{u}^{-}:=\left\{v \in S^{n-1} \mid\langle u, v\rangle \leqq 0\right\}$.

K. Zindler [47], Satz 27, proved, under some regularity hypotheses, and P. C. Hammer and T. J. Smith [19], Theorem 2.2, proved, in full generality, the following

Theorem 1.1 ([47], Satz 27, [19], Theorem 2.2). Let $K$ be a convex body in the Euclidean plane. Then the following are equivalent.

1. $K$ is centrally symmetric.

2. For any two points $p, q \in$ bd $K$, such that the counterclockwise arcs $p q$, $q p$ of bd $K$ have equal Euclidean lengths, we have that $p$ and $q$ are antipodal points of $K$.

In their proof, Hammer and Smith essentially used the Euclidean property of the norm. However, without proof they announced the following theorem.

Theorem 1.2 ([7], Theorem 2, [4], Theorem 4). Let $\mathbb{M}^{2}$ be a twodimensional Minkowski space, and $K \subset \mathbb{M}^{2}$ be a convex body. Then the following conditions are equivalent.

1. $K$ is centrally symmetric.

2. For any two points $p, q \in$ bd $K$, such that the counterclockwise arcs $p q$ and $q p$ of bd $K$ have equal Minkowski lengths, we have that $p$ and $q$ are antipodal points of $K$.

3. For a dense set of points $p \in \operatorname{bd} K$, and the point $q \in \operatorname{bd} K$ such that the counterclockwise arcs $p q$ and $q p$ of bd $K$ have equal Minkowski lengths, we have that $p$ and $q$ are antipodal points of $K$.

We remark that Y. D. Chai and Y. I. Kim [7], Theorem 2, proved our Theorem 1.2 only in a special case. Then G. Averkov [4], Theorem 4, proved Theorem 1.2 in full generality. (Still we remark that in [7], before their Theorem 2, they write "analytic", in their Theorem 2 they write "smooth", but 
their proof shows that they have meant $C^{2}$ and strictly positive Euclidean curvature. Their other extra hypothesis is that $K-K$ is some constant times the unit ball of $\mathbb{M}^{2}$. Moreover, [7], Theorem 2, had in 2. a converse implication. However, since the hypotheses of [7], Theorem 2, imply smoothness and strict convexity of $K$, our implication and the converse implication in 2. of our Theorem 1.2 are equivalent.)

On the other hand, R. Schneider extended Theorem 1.1 in another direction, namely to $\mathbb{R}^{d}$. In [38], as Satz 4.4, he proved the following

Theorem 1.3 ([38], Satz 4.4). Let $K \subset \mathbb{R}^{d}$ be a convex body. Suppose that the shadow boundary of $K$, with respect to parallel illumination from each direction $u \in S^{d-1}$, halves the Euclidean surface area of $K$ (in the sense of Definition 1.4, putting $f=1$ there). Then $K$ is centrally symmetric.

Now we recall from [40], p. 203, the definitions of the curvature measures and area measures of a convex body $K \subset \mathbb{R}^{d}$. For this we introduce some notations, following [40]. Let $x \in \mathbb{R}^{d} \backslash K$. Then there is a unique point of $K$ closest to $x$, which we denote by $p(K, x)$. We write $d(K, x):=|x-p(K, x)|$, and $u(K, x):=(x-p(K, x)) / d(K, x)$, with $|\cdot|$ denoting again the Euclidean norm.

For $B \subset \mathbb{R}^{d}$ a Borel set and $\varrho>0$ we consider the Lebesgue measure of the set $\left\{x \in \mathbb{R}^{d} \mid 0<d(K, x) \leqq \varrho, p(K, x) \in B\right\}$; it is of the form

$$
\frac{1}{d} \sum_{k=0}^{d-1} \varrho^{d-k}\left(\begin{array}{l}
d \\
k
\end{array}\right) C_{k}(K, B),
$$

with $C_{k}(K, \cdot)$, for $0 \leqq k \leqq d-1$, being a finite non-negative Borel measure on $\mathbb{R}^{d}$ (concentrated on bd $K$ ). It is called the $k$-th curvature measure of $K$.

For $B \subset S^{d-1}$ a Borel set we consider the Lebesgue measure of the set $\left\{x \in \mathbb{R}^{d} \mid 0<d(K, x) \leqq \varrho, u(K, x) \in B\right\}$; it is of the form

$$
\frac{1}{d} \sum_{k=0}^{d-1} \varrho^{d-k}\left(\begin{array}{l}
d \\
k
\end{array}\right) S_{k}(K, B),
$$

with $S_{k}(K, \cdot)$, for $0 \leqq k \leqq d-1$, being a finite non-negative Borel measure on $S^{d-1}$. It is called the $k$-th area measure of $K$. For $k=d-1$ this coincides with the surface area measure of $K$, while for $k=0$ it is, independent of $K$, the Lebesgue measure on $S^{d-1}$, cf. [40], Theorem 4.2.5.

Concerning curvature measures, Schneider (for $k=d-1$ see [38], Satz 4.2, for the general case we refer to [39], Theorem 9.6) proved, with $H_{u}^{+}:=$ $\left\{x \in \mathbb{R}^{d} \mid\langle x, u\rangle \leqq 0\right\}$ and $H_{u}^{-}:=\left\{x \in \mathbb{R}^{d} \mid\langle x, u\rangle \leqq 0\right\}$ for $u \in S^{d-1}$, the following 
Theorem 1.4 ([38], Satz 4.2, [39], Theorem 9.6). Let $K \subset \mathbb{R}^{d}$ be a convex body with $O \in \operatorname{int} K$, and let $k$ be an integer with $0 \leqq k \leqq d-1$. Suppose that for each $u \in S^{d-1}$ we have

$$
C_{k}\left(K, H_{u}^{+}\right)=C_{k}\left(K, H_{u}^{-}\right) .
$$

Then $K$ is $O$-symmetric.

The aim of the present paper is, on the one hand, to give a common generalization of Theorems 1.2 and 1.3, for $d$-dimensional Minkowski spaces. In fact, we use little from the definition of Minkowskian surface area(s). We may measure "surface area" via any even Borel function $\varphi: S^{d-1} \rightarrow \mathbb{R}$, for a convex body with Euclidean surface area measure $d S_{K}(u)$, with $\varphi(u)$ being $d S_{K}(u)$-almost everywhere non-0, by the formula $B \mapsto \int_{B} \varphi(u) d S_{K}(u)$ (supposing that $\varphi$ is integrable with respect to $d S_{K}(u)$ ), for $B \subset S^{d-1}$ a Borel set.

On the other hand, observe that Theorems 1.3 and 1.4 of Schneider are somewhat analogous (or, in some sense, dual) to each other. In Theorem 1.4 one cuts bd $K$ into two parts by linear $(d-1)$-subspaces, allowing $k$ to vary from 0 to $d-1$. In Theorem 1.3 this "cutting by linear $(d-1)$-subspaces" is made for $S^{d-1}$, i.e., on the spherical image of bd $K$ - at least for bd $K$ strictly convex - (i.e., one cuts bd $K$ into two parts by shadow boundaries with respect to parallel illumination). However, Theorem 1.3 is related to the surface area measure $d S_{K}(u)$ of $K$, that is $S_{k}(K, \cdot)$, for $k=d-1$. Analogously to Theorem 1.4, we will extend Theorem 1.3 to the cases $1 \leqq k \leqq d-2$. Moreover, denoting $S_{k}(K, \cdot)$ also by $d S_{K, k}(u)$, we may consider, rather than $d S_{K, k}(u)$, more generally finite signed Borel measures $B \mapsto \int_{B} \varphi(u) d S_{K, k}(u)$, where $B \subset S^{d-1}$ is a Borel set, $\varphi: S^{d-1} \rightarrow \mathbb{R}$ is an even Borel function, integrable with respect to $d S_{K, k}(u)$, and being $d S_{K, k}(u)$-almost everywhere non-0. The conclusion will remain the same.

Finally in this section we want to mention some similar characterizations of centrally symmetric convex bodies in Euclidean space, namely [47], [42], [19], [12], and [26], see also [7] and [38] mentioned before, and Section 4 of the survey [21]. The results in these papers are related to our paper by the chosen methods and/or by their geometric nature.

We stress only one characterization, that is a perfect analogue of Theorem 1.1. K. Zindler [47], Satz 26, proved under some regularity hypotheses and P. C. Hammer and T. J. Smith [19], Theorem 2.4, proved in full generality the following. A planar convex body $K$ is centrally symmetric if and only if for each chord $p q$ of $K$, halving the area of $K$, we have that $p$ and $q$ are antipodal points of $K$. Observe that the area, half-perimeter, and total curvature of $K$ are just the quermassintegrals of $K$. Thus there remains one more analogous question. What can be said about $K$ if the set of perimeter-halving chords of $K$ coincides with the set of area-halving chords 
of $K$. Clearly, central symmetry of $K$ implies the italicized property. However, K. Zindler [47], Satz 25, gave an explicit example of a not centrally symmetric $K$ with the italicized property. Later H. Auerbach [3], $\S 6$, determined the set of all planar convex bodies $K$ with the italicized property. (In fact, he stated this for the set of all so called "convex (Z)-curves". However, in pp. $122-123$ he stated that the italicized property of $K$ implies that the boundary of $K$ is a convex (Z)-curve, while in p. 134 he stated that if the boundary of $K$ is a convex (Z)-curve then $K$ has the italicized property.)

\section{Spherical harmonics}

We recall that we work in $d$-dimensional Euclidean space $\mathbb{R}^{d}, d \geqq 2$. Spherical harmonics are $d$-dimensional generalizations of the trigonometric functions $\cos (n \varphi)$, $\sin (n \varphi)$ with $\varphi \in[0,2 \pi]$ (or we can say $\varphi \in S^{1}$ ). Standard references are [15], [20], [32], [41], [10], [45], Ch. IX, [46], [2], Ch. 9, and, for $d=3$ in more detail, [35]. Further references, with geometrical applications, are, e.g., [13], Kap. 2, [1], [33], Cor. 1.31, [44], [5], § 23, Anhang, [34], $\S 4,[36],[37],[38],[8],[12],[16]$, Appendix C, [26], and also the survey [17]. Also we refer to the books [40], pp. 428-432, and [18], which also contain ample further bibliography. Some further papers in geometry or analysis, related to the topic of our paper, are [14], [24], and [27].

A polynomial $f: \mathbb{R}^{d} \rightarrow \mathbb{R}$ is harmonic if $\sum_{i=1}^{d}\left(\partial / \partial x_{i}\right)^{2} f=0$. (This is invariant under the choice of an orthonormal base.) For an integer $n \geqq 0$ a spherical harmonic (of degree $n$ ) in $d$ dimensions is the restriction of a homogeneous harmonic polynomial $f: \mathbb{R}^{d} \rightarrow \mathbb{R}$ (of degree $n$ ) to $S^{d-1}$. (Since $d$ will be fixed, later we will not refer to the dimension.) The spherical harmonics of degree $n$ form a finite dimensional vector space of positive dimension. Choosing from this vector space an orthonormal base $\left\{Y_{n, i} \mid 1 \leqq i \leqq N(d, n)\right\}$ (orthonormality meant in the space $L^{2}\left(S^{d-1}\right)$, for the Lebesgue measure on $\left.S^{d-1}\right)$, their union for each integer $n \geqq 0$ is a complete orthonormal system in $L^{2}\left(S^{d-1}\right)$. Thus each $f \in L^{2}\left(S^{d-1}\right)$ has a Fourier expansion $\sum_{n=0}^{\infty}\left(\sum_{i=1}^{N(d, n)} c_{n i} Y_{n i}\right)$. Here we will write $\sum_{i=1}^{N(d, n)} c_{n i} Y_{n i}=Y_{n}(f)$. Thus the Fourier expansion of $f$ is $\sum_{n=0}^{\infty} Y_{n}(f)$.

\section{The new results for $d$-dimensional Minkowski spaces}

Let us consider a $d$-dimensional Minkowski space $\mathbb{M}^{d}$, with underlying Euclidean space $\mathbb{R}^{d}$. We will considerably weaken the requirement on the Minkowskian surface area measure. Rather than $\int_{B} d S_{K}(u) / f\left(K \cap u^{\perp}\right)$, we 
will consider the signed Borel measure $B \mapsto \int_{B} \varphi(u) d S_{K}(u)$ - where $B \subset$ $S^{d-1}$ is a Borel set - that satisfies the following two conditions.

a) $\varphi: S^{d-1} \rightarrow \mathbb{R}$ is an even Borel measurable function, with

$$
\int_{S^{d-1}}|\varphi(u)| d S_{K}(u)<\infty,
$$

i.e., the total variation of the above signed Borel measure being finite, and

b) $\varphi(u) \neq 0$ for $d S_{K}(u)$-almost every $u \in S^{d-1}$.

Furthermore, we will use the notations $S_{u}^{+}$and $S_{u}^{-}$introduced in Definition 1.4, and $d u$ is the Lebesgue measure on $S^{d-1}$.

Theorem 3.1. Let $K$ be a convex body in $\mathbb{R}^{d}$, let $d S_{K}(u)$ be the Euclidean surface area measure of $K$, and $\varphi: S^{d-1} \rightarrow \mathbb{R}$ be a function satisfying a) and b) above. Then the following statements are equivalent.

A) The body $K$ is centrally symmetric.

B) The equality

$$
\int_{S_{u}^{+}} \varphi(u) d S_{K}(u)=\int_{S_{u}^{-}} \varphi(u) d S_{K}(u)
$$

holds for every direction $u \in S^{d-1}$.

C) Equality (1) holds for du-almost every direction $u \in S^{d-1}$.

D) Equality (1) holds for du-almost every direction $u \in S^{d-1}$ among those directions $u$, for which the shadow boundary of $K$ with respect to parallel illumination from direction $u$ is sharp.

DeFinition 3.1. The shadow boundary of the convex body $K \subset \mathbb{R}^{d}$ with respect to parallel illumination from direction $u \in S^{d-1}$ halves the $k$-th area measure $d S_{K, k}(u)$ of $K$, with $k$ an integer satisfying $1 \leqq k \leqq d-2$, if

$$
\int_{S_{u}^{+}} d S_{K, k}(u)=\int_{S_{u}^{-}} d S_{K, k}(u) .
$$

Using this definition, we state

THEOREM 3.2. Let $k$ be an integer with $1 \leqq k \leqq d-2$, and $K \subset \mathbb{R}^{d}$ be $a$ convex body. Suppose that the shadow boundary of $K$, with respect to parallel illumination from each direction $u \in S^{d-1}$, halves the $k$-th area measure of $K$. Then $K$ is centrally symmetric. 
As Theorem 3.1 is a generalization of Theorem 1.3 of Schneider, we will also generalize Theorem 3.2 in a similar way.

THEOREM 3.3. Let $k$ be an integer with $1 \leqq k \leqq d-2$, and $K \subset \mathbb{R}^{d}$ be a convex body. Suppose that the hypotheses a) and b) before Theorem 3.1 hold, for some $\varphi: S^{d-1} \rightarrow \mathbb{R}$, with $d S_{K, k}(u)$ rather than $d S_{K}(u)$. Then the statements $\mathrm{A}), \mathrm{B}), \mathrm{C}), \mathrm{D})$ of Theorem 3.1 remain equivalent, if $d S_{K}(u)$ is everywhere replaced by $d S_{K, k}(u)$.

\section{The proofs of our theorems}

For the proofs we will need four statements: the Funk-Hecke theorem, and three statements taken essentially from [38]. These four statements are our next four lemmas.

We recall the Funk-Hecke theorem as stated in [41], Theorem 3. The Gegenbauer polynomials $C_{n}(t)$ ( $n \geqq 0$ is an integer) form a sequence of non-0 polynomials of $n$-th degree, satisfying the orthogonality relations

$$
\int_{-1}^{1} C_{n}(t) C_{m}(t)\left(1-t^{2}\right)^{(d-3) / 2} d t=0 .
$$

We have $C_{n}(1) \neq 0$ (cf. [41], (3), where also the value of $C_{n}(1)$ is given; however, we will not need its concrete value).

Lemma A (Funk-Hecke theorem, see [13], Kap. 2, [14], [15] and [20]). Let $F:[-1,1] \rightarrow \mathbb{R}$ be Borel measurable, with

$$
\int_{-1}^{1}|F(t)|\left(1-t^{2}\right)^{(d-3) / 2} d t<\infty
$$

and let $H_{n}$ be a spherical harmonic of degree $n$ on $S^{d-1}$, where $n \geqq 0$ is an integer. Then $H_{n}(u)$ is an eigenfunction of the integral transformation $I$ given by

$$
(I(H))(u):=\int_{S^{d-1}} F(\langle u, v\rangle) H(v) d v,
$$


where $d v$ is the usual Lebesgue measure on $S^{d-1}$. Moreover, for each $u \in$ $S^{d-1}$, the integral $\int_{S^{d-1}} F(\langle u, v\rangle) H_{n}(v) d v$ equals

$V_{d-2}\left(S^{d-2}\right) C_{n}(1)^{-1} \cdot \int_{-1}^{1} F(t) C_{n}(t)\left(1-t^{2}\right)^{(d-3) / 2} d t \cdot H_{n}(u)=: \lambda_{d, n}(F) H_{n}(u)$.

Here $V_{d-2}\left(S^{d-2}\right)$ is the usual $(d-2)$-dimensional Lebesgue measure of $S^{d-2}$ $\left(V_{0}\left(S^{0}\right)\right.$ meant as 2$)$.

The formulation of the following lemma is slightly more general than in [38] and [18], but the proof is essentially the same. (In [38] this was used for $F$ continuous with the exception of finitely many points, where the left and right hand side limits existed. In [18] it was observed that the same proof is valid for $F$ being any bounded Borel function. Here we give a variant using the largest feasible class of functions, for which still essentially the same proof goes through.)

Lemma B ([38], Satz 3.1, cf. also [18], Lemma 3.4.4). Let $\mu$ be a finite signed Borel measure on $S^{d-1}$. Let $F:[-1,1] \rightarrow \mathbb{R}$ be a Borel function, such that the function $(u, v) \mapsto F(\langle u, v\rangle)$ is $d u \times d|\mu|(v)$-integrable (i.e., $\int_{S^{d-1} \times S^{d-1}}|F(\langle u, v\rangle)|(d u \times d|\mu|(v))<\infty$, where $d u$ is Lebesgue measure on $S^{d-1}$ and $|\mu|$ is the total variation of $\left.\mu\right)$, and let for du-almost every $u \in S^{d-1}$

$$
\int_{S^{d-1}} F(\langle u, v\rangle) d \mu(v)=0
$$

Further let $n \in\{0,1, \ldots\}$, and $\lambda_{d, n}(F)$ from Lemma $\mathrm{A}$ be not equal to 0 . Then for every spherical harmonic $H_{n}$ of degree $n$ we have

$$
\int_{S^{d-1}} H_{n}(u) d \mu(u)=0
$$

Proof. By Fubini's theorem for $d u$-almost every $u \in S^{d-1}$ the function $v \mapsto F(\langle u, v\rangle)$ is $d \mu(v)$-integrable (i.e., is $d|\mu|(v)$-integrable). Hence for $d u$ almost every $u$ the hypothesis

$$
\int_{S^{d-1}} F(\langle u, v\rangle) d \mu(v)=0
$$


makes sense. By hypothesis we may consider the integral

$$
\int_{S^{d-1} \times S^{d-1}} F(\langle u, v\rangle) H_{n}(u)(d u \times d \mu(v)),
$$

where $n \in\{0,1, \ldots\}$ and $H_{n}(u)$ is any spherical harmonic on $S^{d-1}$ of order $n$. This integral can be evaluated by Fubini's theorem, by first integrating with respect to $u$, and then with respect to $v$, or in the converse order. In the second case, we obtain by hypothesis at the first integration for $d u$-almost every $u \in S^{d-1}$ the value 0 , as the value of the first integral. Then, integrating it with respect to $d u$, we obtain the value 0 , as the value of the double integral. Integration, first with respect to $d u$, and then with respect to $d \mu(v)$, and the Funk-Hecke theorem (Lemma A) give

$$
0=\int_{S^{d-1}}\left(\int_{S^{d-1}} F(\langle u, v\rangle) H_{n}(u) d u\right) d \mu(v)=\int_{S^{d-1}} \lambda_{d, n}(F) H_{n}(v) d \mu(v) .
$$

By hypothesis $\lambda_{d, n}(F) \neq 0$, which implies the claim of the lemma.

Lemma C ([38], p. 58 (2.), cf. also [18], Lemma 3.4.6). In Lemma A, let $F:[-1,1] \rightarrow \mathbb{R}$ be the characteristic function of $[0,1]$. Then the eigenvalues $\lambda_{d, n}(F)$ from Lemma A satisfy $\lambda_{d, n}(F)=0$ for $n \geqq 2$ even, and $\lambda_{d, n}(F) \neq 0$ for $n=0$ and for $n$ odd.

For the function $F$ in Lemma $C$ the values of $\lambda_{d, n}(F)$ are given by [18], Lemma 3.4.6; however, we will not need their concrete values here, only whether they are 0 or not.

The formulation of Lemma D (like of Lemma B) is slightly more general than in [38] and [18].

Lemma D ([38], Korollar 3.2, cf. also [18], Proposition 3.4.11). Let $\mu$ be a finite signed Borel measure on $S^{d-1}$, such that $\mu\left(S_{u}^{+}\right)=0$ for du-almost every $u \in S^{d-1}$, where $d u$ is the Lebesgue measure on $S^{d-1}$. Then $\mu$ is even (i.e., for any Borel set $B \subset S^{d-1}$, we have $\mu(B)=\mu(-B)$ ).

Proof. The hypothesis can be written as $\int_{S^{d-1}} F(\langle u, v\rangle) d \mu(v)=0$ for $d u$-almost all $u \in S^{d-1}$, with $F$ as defined in Lemma C. By Lemma B for any $n \in\{0,1, \ldots\}$ with $\lambda_{d, n}(F) \neq 0$ we have for any spherical harmonic $H_{n}$ of degree $n$

$$
\int_{S^{d-1}} H_{n}(u) d \mu(u)=0 .
$$


Thus, by Lemma C, (2) surely holds for all odd $n \in\{0,1, \ldots\}$.

To show that $\mu$ is even, let $B \subset S^{d-1}$ be a Borel set. We have to show that $\mu(B)-\mu(-B)=0$. However, the set function $B \mapsto \nu(B):=\mu(B)-\mu(-B)$ is an odd set function, i.e., $\nu(-B)=-\nu(B)$ for all Borel sets $B \subset S^{d-1}$. Then certainly $\int_{S^{d-1}} f(u) d \nu(u)=0$ for all even functions, in particular, for any even $n$, for any spherical harmonic $H_{n}$ of degree $n$ ( $H_{n}$ being homogeneous of $n$-th degree, thus being an even function).

However, also for any odd $n$, and any spherical harmonic $H_{n}$ of degree $n$ (that is again homogeneous of $n$-th degree, thus now is an odd function), we have

$$
\begin{aligned}
& \int_{S^{d-1}} H_{n}(u) d \nu(u)=\int_{S^{d-1}} H_{n}(u) d \mu(u)-\int_{S^{d-1}} H_{n}(u) d \mu(-u) \\
& =\int_{S^{d-1}} H_{n}(u) d \mu(u)-\int_{S^{d-1}} H_{n}(-u) d \mu(u)=2 \int_{S^{d-1}} H_{n}(u) d \mu(u)=0
\end{aligned}
$$

by (2) that is valid for any odd $n$; cf. the sentence following (2).

Thus, for any $n \in\{0,1, \ldots\}$, and any spherical harmonic $H_{n}$ of degree $n$, we have

$$
\int_{S^{d-1}} H_{n}(u) d \nu(u)=0 .
$$

Then Lemma 3.2 from [38] (cf. also [18], Proposition 3.2.8) implies $\nu=0$, i.e., for any Borel set $B \subset S^{d-1}$ we have $\mu(B)-\mu(-B)=0$, i.e., $\mu$ is even.

Proof of Theorem 3.1. Of course it is sufficient to prove D) $\Rightarrow A$ ) in Theorem 3.1.

I) Although this is not necessary, first we will show $\mathrm{D}) \Rightarrow \mathrm{A}$ ) for $K$ a convex $d$-polytope, due to the extreme simplicity of the proof in this case. Let $K$ have facets $F_{i}$, with outer unit normals $u_{i}$, and $(d-1)$ volumes $V_{d-1}\left(F_{i}\right), i=1, \ldots, m$.

The shadow boundary of $K$ with respect to parallel illumination from the direction $u \in S^{d-1}$ is sharp exactly when $u \in S^{d-1} \backslash \bigcup_{i=1}^{m} u_{i}^{\perp}$. If $u \in S^{d-1} \backslash \bigcup_{i=1}^{m} u_{i}^{\perp}$, then the set of illuminated facets and the respective shadow boundary are locally constant. Since D) is satisfied for almost all directions $u$ in $S^{d-1} \backslash \bigcup_{i=1}^{m} u_{i}^{\perp}$, then certainly it is satisfied for a dense subset of this set. Hence, by local constantness of $\sum_{\left\langle u, u_{i}\right\rangle>0} V_{d-1}\left(F_{i}\right) \varphi\left(u_{i}\right)$, for each $u \in S^{d-1} \backslash \bigcup_{i=1}^{m} u_{i}^{\perp}$ we have

$$
\sum_{\left\langle u, u_{i}\right\rangle>0} V_{d-1}\left(F_{i}\right) \varphi\left(u_{i}\right)=\frac{1}{2} \sum_{i=1}^{m} V_{d-1}\left(F_{i}\right) \varphi\left(u_{i}\right),
$$


so it is independent of $u \in S^{d-1} \backslash \bigcup_{i=1}^{m} u_{i}^{\perp}$.

For $i, j \in\{1, \ldots, m\}$ we say that $u_{i}, u_{j}$ are equivalent if $u_{j}= \pm u_{i}$. Then the equivalence classes have one or two elements. If an equivalence class has two elements, say $u_{i}$ and $u_{j}$, we may have $V_{d-1}\left(F_{i}\right)=V_{d-1}\left(F_{j}\right)$, or $V_{d-1}\left(F_{i}\right) \neq V_{d-1}\left(F_{j}\right)$.

Let us choose a $u_{i}$. Its equivalence class $C_{i}$ is either $\left\{u_{i},-u_{i}\right\}$, or $\left\{u_{i}\right\}$. In the first case $S^{d-1} \cap u_{i}^{\perp}=S^{d-1} \cap\left(-u_{i}\right)^{\perp}$. Let us choose $v \in\left(S^{d-1} \cap\right.$ $\left.u_{i}^{\perp}\right) \backslash \bigcup_{u_{j} \in\left\{u_{1}, \ldots, u_{m}\right\} \backslash C_{i}} u_{j}^{\perp}$. Then, for $|\varepsilon|$ sufficiently small, $v_{\varepsilon}=(v+$ $\left.\varepsilon u_{i}\right) /\left|v+\varepsilon u_{i}\right| \in S^{d-1} \backslash \bigcup_{j=1}^{m} u_{j}^{\perp}$. We have

$$
0=\lim _{0<\varepsilon \rightarrow 0} \sum_{\left\langle v_{\varepsilon}, u_{j}\right\rangle>0} V_{d-1}\left(F_{j}\right) \varphi\left(u_{j}\right)-\lim _{0>\varepsilon \rightarrow 0} \sum_{\left\langle v_{\varepsilon}, u_{j}\right\rangle>0} V_{d-1}\left(F_{j}\right) \varphi\left(u_{j}\right),
$$

where the limits in question evidently exist. The last expression equals $V_{d-1}\left(F_{i}\right) \varphi\left(u_{i}\right)-V_{d-1}\left(F_{j}\right) \varphi\left(u_{j}\right)$, if $\left|C_{i}\right|=2$, where $u_{j}=-u_{i}$, or $V_{d-1}\left(F_{i}\right) \varphi\left(u_{i}\right)$, if $\left|C_{i}\right|=1$, respectively. By hypothesis $\varphi\left(u_{i}\right) \neq 0$, so the second case cannot occur. In the first case $0=\left(V_{d-1}\left(F_{i}\right)-\right.$ $\left.V_{d-1}\left(F_{j}\right)\right) \varphi\left(u_{i}\right)$, so $V_{d-1}\left(F_{i}\right)=V_{d-1}\left(F_{j}\right)$. Therefore all equivalence classes have two elements, $u_{i}$ and $u_{j}=-u_{i}$, say, and $V_{d-1}\left(F_{i}\right)=$ $V_{d-1}\left(F_{j}\right)$. Thus $d S_{K}(u)=d S_{K}(-u)$, and $d S_{K}(u)$ or $d S_{K}(-u)$ is the surface area measure of $K$, or $-K$, respectively. Hence $-K$ is a translate of $K$ (cf. [40], Theorem 7.2.1), i.e., $K$ is centrally symmetric.

II) Now let $K \subset \mathbb{R}^{d}$ be an arbitrary convex body. By [11] (see also [23]), for $d u$-almost every direction $u \in S^{d-1}$ ( $d u$ is Lebesgue measure on $S^{d-1}$ ), we have that the shadow boundary of $K$ with respect to parallel illumination from the direction $u$ is sharp. This shows that $\mathrm{D}$ ) implies C). Therefore it remains to show that C) implies A), which will be done in the following.

We have that $\varphi$ is an even Borel function, non- $0 d S_{K}(u)$-almost everywhere. The set $\varphi^{-1}(0) \subset S^{d-1}$ is $O$-symmetric; we change the value of $\varphi$ there to 1 . The function thus obtained is an even Borel function, nowhere 0 , and is $d S_{K}(u)$-almost everywhere equal to $\varphi$. From now on, we will for simplicity use the same notation $\varphi$ for the function obtained by this changing.

We have that $d S_{K}(u)$ is a finite non-negative measure on the Borel sets of $S^{d-1}$. Let us denote this measure by $\mu$. Then the set function $\nu$, defined on the Borel sets $B$ of $S^{d-1}$ by $\nu(B)=\int_{B} \varphi(u) d \mu(u)$, is a finite signed measure on the Borel sets of $S^{d-1}$. It has total variation $|\nu|$, a finite non-negative Borel measure on $S^{d-1}$, given by $|\nu|(B)=\int_{B}|\varphi(u)| d \mu(u)$. Evidently $|\nu|$ is absolutely continuous with 
respect to $\mu$, and, by $\varphi(u) \neq 0$ for each $u \in S^{d-1}$, we have that also $\mu$ is absolutely continuous with respect to $|\nu|$. Hence the Borel sets $B \subset S^{d-1}$ with $\mu(B)=0$ coincide with the Borel sets $B \subset S^{d-1}$ with $|\nu|(B)=0$.

By the absolute continuities of $\mu$ and $|\nu|$ with respect to each other, there exists the Radon-Nikodým derivative $d \nu / d \mu$, defined $\mu$-almost everywhere, belonging to $L^{1}(\mu)$, and equal to $\varphi, \mu$-almost everywhere, and the Radon-Nikodým derivative $d \mu / d \nu$, defined $|\nu|$-almost everywhere (i.e., $\mu$-almost everywhere), belonging to $L^{1}(|\nu|)$. We have $\mu$-almost everywhere $1=d \mu / d \mu=(d \mu / d \nu)(d \nu / d \mu)=(d \mu / d \nu) \varphi$, see [9], p. 122, Exercise 1. Hence $d \mu / d \nu=\varphi^{-1}$ holds $\mu$-almost everywhere. In other words, for each Borel set $B \subset S^{d-1}$, we have $\mu(B)=\int_{B} \varphi^{-1}(u) d \nu(u)$.

By C) of our theorem we have for $d u$-almost every $u \in S^{d-1}$ that $\nu\left(S_{u}^{+}\right)=\nu\left(S_{u}^{-}\right)$. Let $\nu^{\prime}$ be the finite signed Borel measure on $S^{d-1}$ defined by $\nu^{\prime}(B)=\nu(-B)$. Then, by the equality in the last but one sentence, we have $\nu\left(S_{u}^{+}\right)=\nu^{\prime}\left(S_{u}^{+}\right)$, i.e., $\left(\nu-\nu^{\prime}\right)\left(S_{u}^{+}\right)=0$, for $d u$ almost every $u \in S^{d-1}$. Observe that the finite signed Borel measure $\nu-\nu^{\prime}$ on $S^{d-1}$ is odd (i.e., for any Borel set $B \subset S^{d-1}$, we have $\left.\left(\nu-\nu^{\prime}\right)(B)=-\left(\nu-\nu^{\prime}\right)(-B)\right)$. On the other hand, from the last but one sentence and Lemma $\mathrm{D}$ we have that $\nu-\nu^{\prime}$ is even. Hence $\nu-\nu^{\prime}=0$, i.e., $\nu(B)=\nu^{\prime}(B)=\nu(-B)$ for each Borel set $B \subset S^{d-1}$. Thus $\nu$ is even. Since $\varphi^{-1}$ is an even function, for any Borel set $B \subset S^{d-1}$ we have

$\mu(B)=\int_{B} \frac{d \mu}{d \nu}(u) d \nu(u)=\int_{B} \varphi^{-1}(u) d \nu(u)=\int_{-B} \varphi^{-1}(u) d \nu(u)=\mu(-B)$,

i.e., $\mu$ is an even measure. In other notation, we have $d S_{K}(u)=$ $d S_{K}(-u)$. Here the left, or right hand side is the surface area measure of $K$, or $-K$, respectively. From their equality we have that $-K$ is a translate of $K$ (cf. [40], Theorem 7.2.1), i.e., $K$ is centrally symmetric.

Proof of Theorems 3.2 And 3.3. We will prove Theorem 3.3, and then Theorem 3.2 follows as a special case of it.

For Theorem 3.3 we proceed as in the proof of Theorem 3.1, Part II. We get that $d S_{K, k}(u)$ is an even measure, or, in other notation, that $d S_{K, k}(u)=$ $d S_{K, k}(-u)$. Here the left, or right hand side is the $k$-th area measure of $K$, or $-K$, respectively. From their equality we have that $-K$ is a translate of $K$ (cf. [40], Corollary 7.2.5), i.e., $K$ is centrally symmetric. 


\section{Remarks}

REMARK 5.1. In Theorem 1.2 the hypothesis was roughly, that equal Minkowskian complementary arc lengths imply equal angular rotation of the counterclockwise tangent vectors, at both of the arcs, i.e., an angular rotation $\pi$. Our Theorem 3.1 was the other way round: roughly, we considered two illuminated parts of the boundary, from opposite directions, and required them to have equal Minkowskian measure. However, Theorem 3.1 implies Theorem 1.2. As we will see, for $K$ smooth and strictly convex, this will follow almost immediately. For the general case, by not difficult arguments, we will show that maximal segments in bd $K$, or non-smooth points of bd $K$ with given cone of outer normals, occur in centrally symmetric pairs, respectively. Moreover, the map assigning to $p \in \mathrm{bd} K$ the point $o(p)$ such that the counterclockwise $\operatorname{arcs} p o(p), o(p) p$ have equal Minkowskian lengths, respects these symmetries. Then the general case will follow easily.

In fact, we will show that a bit more general theorem than Theorem 1.2 follows from our Theorem 3.1. The concise sketch of the proof of Theorem 1.2 from Theorem 3.1 in the preceding paragraph will be the guideline for the proof of the more general Theorem 5.1. We will use the notations introduced before Theorem 3.1, for $d=2$. For $\varphi: S^{1} \rightarrow \mathbb{R}$ we assume a) before Theorem 3.1, and the following strengthening of b) before Theorem 3.1:

b $\left.^{\prime}\right) \varphi(u)>0$ for $d S_{K}(u)$-almost every $u \in S^{1}$. We will write $x$ for a variable point on the boundary of a convex body $K \subset \mathbb{R}^{2}$, further $n(x)$ for the unit outer normal to $K$ at $x$ (provided it is uniquely determined), and $d s$ for the Euclidean arc element. We have that $n(x)$ is uniquely determined, except for an at most countably infinite set $E \subset \mathrm{bd} K$. Moreover, $n(x)$ is continuous on (bd $K) \backslash E$, hence, is a Borel function, on the Borel set (bd $K) \backslash E$. When writing $\int_{B} \varphi(n(x)) d s$, for $B \subset$ bd $K$ a Borel set, we mean $\int_{B \backslash E} \varphi(n(x)) d s$, which is well defined.

Theorem 5.1. Let $K$ be a convex body in $\mathbb{R}^{2}$, let $d S_{K}(u)$ be the Euclidean surface area measure of $K$, and $\varphi: S^{1} \rightarrow \mathbb{R}$ be a function satisfying a) before Theorem 3.1 and $\mathrm{b}^{\prime}$ ) before this theorem. Then the following conditions are equivalent.

1. $K$ is centrally symmetric.

2. For any two points $p, q \in \mathrm{bd} K$, such that the integrals $\int \varphi(n(x)) d s$ taken on the closed counterclockwise arcs $p q$ and $q p$ of bd $K$ are equal, we have that $p$ and $q$ are antipodal points of $K$.

3. For a dense set of points $p \in \mathrm{bd} K$, and the point $q \in \mathrm{bd} K$ such that the integrals $\int \varphi(n(x)) d s$ taken on the closed counterclockwise arcs $p q$ and $q p$ of $\operatorname{bd} K$ are equal, we have that $p$ and $q$ are antipodal points of $K$.

We remark that Averkov's proof of [4], Theorem 4, readily gives the implication $2 . \Rightarrow 1$. in this more general Theorem 5.1 as well. 
Proof of Theorem 5.1. Of course, it is sufficient to show $3 . \Rightarrow 2 . \Rightarrow 1$. 3 . $\Rightarrow 2$. follows from a trivial continuity consideration.

We turn to show 2. $\Rightarrow 1$. Like in the proof of Theorem 3.1, we may and will assume $\varphi(u)>0$ for each $u \in S^{1}$ (by changing its values to 1 on $\left.\varphi^{-1}((-\infty, 0])\right)$. We retain the notation $o(p) \in \operatorname{bd} K$ for $p \in \operatorname{bd} K$ for that unique point of bd $K$, for which the integrals $\int \varphi(n(x)) d s$ taken on the closed counterclockwise $\operatorname{arcs} p o(p)$ and $o(p) p$ are equal (its existence and unicity are implied by the positivity of $\varphi$ ). Observe $o(o(p))=p$ for $p \in \operatorname{bd} K$. The point $o(p)$ depends continuously on $p$, and moving $p$ counterclockwise along bd $K$, the point $o(p)$ also moves counterclockwise (strictly) along bd $K$. For a point $p \in \operatorname{bd} K$, the set of points of $K$ antipodal to $p$ will be denoted by $A(p)$. This is a closed arc on bd $K$ (for $p$ a smooth point of bd $K$ it is a closed segment or a point). So 2 . of Theorem 5.1 says that $o(p) \in A(p)$.

Now let us suppose that bd $K$ contains a (maximal non-degenerate) segment $\left[p_{1}, p_{2}\right]$, where $p_{2}$ follows $p_{1}$ counterclockwise. Let a relative interior point $p$ of $\left[p_{1}, p_{2}\right]$ move from $p_{1}$ to $p_{2}$, with a uniform Euclidean speed. The point $p$ lies on a unique supporting line of $K$, namely aff $\left[p_{1}, p_{2}\right]$ (aff $A$ denotes the affine hull of the set $A \subset \mathbb{R}^{2}$ ). Then any point of $K$, which is antipodal to $p$, must lie on the other supporting line $L$ of $K$, parallel to aff $\left[p_{1}, p_{2}\right]$. Thus, by moving $p$, the point $o(p)$ moves on the supporting line $L$ of $K$. By the equality of the integrals $\int \varphi(n(x)) d s$ on the closed counterclockwise arcs $p o(p)$ and $o(p) p$, if $p$ moves on $\left[p_{1}, p_{2}\right]$ with a uniform Euclidean speed from $p_{1}$ to $p_{2}$, then $o(p)$ moves on $L$ in the opposite direction as $p$, but with the same uniform Euclidean speed, from $o\left(p_{1}\right)$ to $o\left(p_{2}\right)$. Hence $L$ contains a (maximal) segment on bd $K$, of Euclidean length at least that of $\left[p_{1}, p_{2}\right]$. Now one can change the roles of $\left[p_{1}, p_{2}\right]$ and $K \cap L$. It follows, that $\left[p_{1}, p_{2}\right]$ and $K \cap L$ are parallel segments of the same Euclidean length, and that for $p \in\left[p_{1}, p_{2}\right]$ the point $o(p)$ is the centrally symmetric image of $p$ with respect to the centre of the parallelogram $p_{1} p_{2} o\left(p_{1}\right) o\left(p_{2}\right)$.

Now let us suppose that $p \in \operatorname{bd} K$ is not a smooth point of bd $K$. Let $L_{1}$ and $L_{2}$ be the (different) extreme supporting lines of $K$ at $p$, such that $L_{1}$ is the half-tangent line to bd $K$ if we move from $p$ clockwise, and $L_{2}$ is the halftangent line to bd $K$ if we move from $p$ counterclockwise. Let, for $i=1,2, L_{i}^{\prime}$ denote the supporting line of $K$, parallel to and different from $L_{i}$. Let $L_{1}^{\prime}$ and $L_{2}^{\prime}$ intersect at $q$. Then $K$ lies in one of the four angular domains into which $L_{1}^{\prime}$ and $L_{2}^{\prime}$ divide $\mathbb{R}^{2}$. Further, $K$ has support sets $K \cap L_{1}^{\prime}$ or $K \cap L_{2}^{\prime}$, on $L_{1}^{\prime}$ or $L_{2}^{\prime}$, respectively. First suppose $q \notin K$. Then the support sets $K \cap L_{1}^{\prime}$ and $K \cap L_{2}^{\prime}$ do not contain $q$ either. Let $i=1,2$. If $K \cap L_{i}^{\prime}$ is a point, let $r_{i}^{\prime}$ denote this point. If $K \cap L_{i}^{\prime}$ is a proper segment, let $r_{i}^{\prime}$ denote its endpoint closer to $q$, cf. Fig. 1.

Then consider the open arc $r_{1}^{\prime} r_{2}^{\prime}$ of bd $K$, not containing $p$. This arc is not degenerate, hence it is smooth, with the exception of at most countably infinitely many of its points. Let $x_{1} \neq x_{2}$ be two points of smoothness of this 


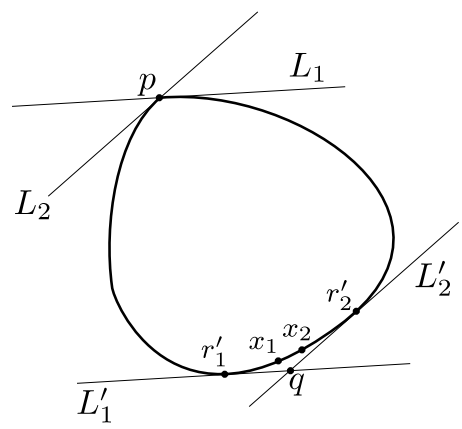

Fig. 1

open arc. Hence the unit outer normals $u_{1}$ or $u_{2}$ of $K$ at $x_{1}$ or $x_{2}$ are in the smaller open arc of $S^{1}$, with endpoints the unit outer normals of $K$, orthogonal to $L_{1}^{\prime}$ or $L_{2}^{\prime}$, at points of $K \cap L_{1}^{\prime}$ or $K \cap L_{2}^{\prime}$, respectively. This implies $A\left(x_{1}\right)=A\left(x_{2}\right)=\{p\}$, hence $o\left(x_{1}\right)=o\left(x_{2}\right)=p$, a contradiction to $x_{1} \neq x_{2}$. This contradiction shows that $q \in K$. Let $L_{1}^{\prime \prime}$ and $L_{2}^{\prime \prime}$ be the extreme supporting lines of $K$ at $q$, where $L_{1}^{\prime \prime}$ or $L_{2}^{\prime \prime}$ is the half-tangent line to bd $K$, if we move from $q$ clockwise, or counterclockwise, respectively. Then the angular domain containing $K$, formed by $L_{1}^{\prime \prime}$ and $L_{2}^{\prime \prime}$, satisfies the following. If we take its image with respect to point reflection at $(p+q) / 2$, then the so obtained angular domain will be a subset of the angular domain with vertex $p$, determined by $L_{1}$ and $L_{2}$, and containing $K$. Now, changing the roles of $p$ and $q$, we obtain that $L_{1}^{\prime}=L_{1}^{\prime \prime}$ and $L_{2}^{\prime}=L_{2}^{\prime \prime}$.

We continue the investigation of the non-smooth point $p \in \mathrm{bd} K$. We have $A(p)=\left(K \cap L_{1}^{\prime}\right) \cup\left(K \cap L_{2}^{\prime}\right)$ and $A(q)=\left(K \cap L_{1}\right) \cup\left(K \cap L_{2}\right)$. We want to show that $o(p)=q$ (and thus $o(q)=p)$. Assume the contrary. We may suppose, without loss of generality, that $o(p) \in\left(K \cap L_{1}^{\prime}\right) \backslash\{q\}$, cf. Fig. 2.

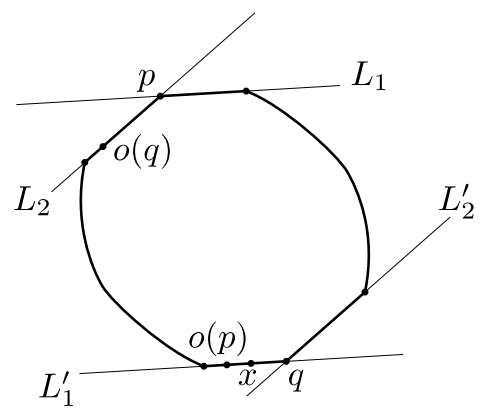

Fig. 2 
Then we have that the integral $\int \varphi(n(x)) d s$ taken on the closed counterclockwise arc $q p$ has a value less than the half of the value of $\int \varphi(n(x)) d s$ taken on bd $K$. Therefore $o(q) \in\left(K \cap L_{2}\right) \backslash\{p\}$. Then $[o(p), q]$ and $[p, o(q)]$ are non-degenerate segments on bd $K$. Now let $x$ be a relative interior point of $[o(p), q]$, which moves from $o(p)$ to $q$ with uniform Euclidean speed. Then, as we have seen above, $o(x)$ moves on a line parallel to $L_{1}^{\prime}$, from $o(o(p))=p$ to $o(q)$. Hence the line spanned by $p$ and $o(q)$, i.e., $L_{2}$, is parallel to $L_{1}^{\prime}$, that is parallel to $L_{1}$. Hence $L_{1}=L_{2}$, a contradiction to the assumption that $p \in \operatorname{bd} K$ is a non-smooth point. This contradiction shows $o(p)=q$ (and thus $o(q)=p)$.

Now we are going to show that statement D) of Theorem 3.1 is satisfied, from which by Theorem 3.1 there will follow the central symmetry of $K$. In fact we will show D) of Theorem 3.1 in the stronger form obtained by replacing " $d u$-almost every" by "every". Let $u \in S^{1}$ be a direction such that the shadow boundary of $K$ with respect to illumination from direction $u$ is sharp. Let this sharp shadow boundary be $\{p, q\}$. Then $p$ and $q$ are antipodal points of $K$.

First suppose that one of $p$ and $q$, e.g., $p$, is not a smooth point of bd $K$. Furthermore, let $L_{1}$ and $L_{2}$ be the two extreme supporting lines of $K$ at $p$, with $L_{1}$ or $L_{2}$ being the half-tangent line to bd $K$, when moving from $p$ along bd $K$ clockwise or counterclockwise, respectively. Let the other supporting lines of $K$, parallel to $L_{1}$ or $L_{2}$, be $L_{1}^{\prime}$ or $L_{2}^{\prime}$, respectively. Let $r$ be the intersection point of $L_{1}^{\prime}$ and $L_{2}^{\prime}$. Then, by what has been said above, $r \in K$ and $L_{1}^{\prime}, L_{2}^{\prime}$ are the extreme supporting lines of $K$ at $r$; moreover, $r=o(p)$ (and $p=o(r)$ ).

We have that $p$ belongs to the shadow boundary of $K$ with respect to parallel illumination from direction $u$, if and only if $u$ lies in the translate through $-p$ of the union of the two opposite closed angular domains determined by $L_{1}$ and $L_{2}$, none containing $K$. Since the pairs $\left\{L_{1}, L_{2}\right\}$ and $\left\{L_{1}^{\prime}, L_{2}^{\prime}\right\}$ are symmetric with respect to $(p+r) / 2$, the above two equivalent valid statements are equivalent also to that $r$ belongs to the shadow boundary of $K$ with respect to parallel illumination from direction $u$. Now $r \neq p$ and this shadow boundary is $\{p, q\} \ni r$. Therefore $o(p)=r=q$. So the values of the integrals $\int \varphi(n(x)) d s$ taken on the closed counterclockwise arcs $p q$ and $q p$ are equal (i.e., (1) from Theorem 3.1 holds).

The remaining case is when both points $p$ and $q$ are smooth points of bd $K$. Then, by hypothesis, $o(p) \in A(p)$. Since there is a unique supporting line $L$ at $p$ to $K$, any point of $K$, antipodal to $p$, must lie in $K \cap L^{\prime}$, where $L^{\prime}$ is the other supporting line of $K$ parallel to $L$. So $q \in A(p)=K \cap L^{\prime}$, and, by hypothesis, $o(p) \in A(p)$. By sharpness of the shadow boundary, $A(p)=$ $K \cap L^{\prime}$ is a point, hence $q=o(p)$, and once more the values of the integrals $\int \varphi(n(x)) d s$ taken on the closed counterclockwise arcs $p q$ and $q p$ are equal (i.e., (1) from Theorem 3.1 holds). 
Remark 5.2. Hypotheses B) and C) of Theorem 3.1 (or Theorem 3.3) are very similar. However, we see no other way of deducing B) from C) than via A) (of course, unless for each $u \in S^{d-1}$ we have

$$
\int_{\left\{v \in S^{d-1} \mid\langle u, v\rangle=0\right\}} d S_{K}(v)=0
$$

for all $u \in S^{d-1}$, when by Lebesgue's dominated convergence theorem

$$
\int_{S_{u}^{+}} \varphi(v) d S_{K}(v)
$$

is a continuous function of $u \in S^{d-1}$; analogously for $\left.d S_{K, k}(u)\right)$. In fact, $\int_{S_{u}^{+}} \varphi(v) d S_{K}(v)$ is not continuous for $K$ a simplex. We remind, that in [26], for another characterization of central symmetry of convex bodies in $\mathbb{R}^{d}$, we had an equation that was satisfied by the nature of the problem for almost all $u \in S^{d-1}$ only.

REMARK 5.3. For special classes of convex bodies in $\mathbb{M}^{d}$, Theorem 3.1 might have consequences that are worth mentioning, too. For example, a convex body $K \subset \mathbb{M}^{d}$ is called a convex body of constant Minkowskian width if the vector sum $K+(-K)$ is a ball in the norm of $\mathbb{M}^{d}$; see [30]. On the other hand, $K \subset \mathbb{M}^{d}$ is said to be a reduced convex body if any proper closed convex subset of $K$ has a smaller minimal width (with distance of different parallel supporting hyperplanes measured in the norm of $\mathbb{M}^{d}$ ); see [25]. Since every centrally symmetric reduced convex body, in particular, every centrally symmetric convex body of constant Minkowskian width in $\mathbb{M}^{d}$ is necessarily a ball of the space (see [25] and [30]), we have that reduced convex bodies, in particular, convex bodies of constant Minkowskian width in $\mathbb{M}^{d}$, having our "halving properties" in the sense of Theorems 3.1 and 3.3 (e.g., with some Minkowskian surface area measure in Theorem 3.1), are necessarily balls in the sense of the norm.

REMARK 5.4. As noticed in [22], in recent years many uniqueness results from convex geometry have been strengthened and formulated as stability statements. Also in certain cases stability versions of characterizations of some special classes of convex bodies can be obtained, see, e.g., [6]. It is possible that using the tools developed in Section 5 of [22], stability versions of characterizations presented in this paper can be obtained, that is, if each shadow boundary of a convex body $K \subset \mathbb{R}^{d}$ nearly halves the Euclidean or Minkowskian surface area of $K$ (or, $k$-th area measure, $1 \leqq k \leqq d-2$ ), then $K$ is nearly centrally symmetric. Similarly one can ask for a stability version 
of Theorem 1.4. Observe that the paper [22] contains some results pointing to this direction.

Acknowledgement. The authors express their gratitude to H. Groemer and R. Schneider for pointing out some references.

\section{REFERENCES}

[1] Alexandroff, A. D., Zur Theorie der gemischten Volumina von konvexen Körpern, II. Neue Ungleichungen zwischen den gemischten Volumina und ihre Anwendungen (Russian, German summary), Mat. Sb., 2 (1937), 1205-1238. Zbl 18,276

[2] Andrews, G. E., Askey, R. and Roy, R., Special Functions. Encyclopedia of Mathematics and its Applications 71, Cambridge University Press, Cambridge, 1999. MR 2000g:33001

[3] Auerbach, H., Sur un problme de M. Ulam concernant l'équilibre des corps flottants, Studia Math., 7 (1938), 121-142. Zbl 18.175.

[4] Averkov, G., On boundary arcs joining antipodal points of a planar convex body, Beiträge Algebra Geom., 47 (2006), no. 2, 489-503. MR 2008f:52004

[5] Blaschke, W., Kreis und Kugel. Zweite durchgesehene verbesserte Auflage, de Gruyter, Berlin, 1956. MR 17,1123

[6] BöröczKY, K., JR., The stability of the Rogers-Shephard inequality and of some related inequalities, Adv. Math., 190 (2005), no. 1, 47-76. MR 2005i:52013

[7] Chai, Y. D. and KIM, Y. I., Curves of constant relative breadth, Kyungpook Math. J., 37 (1997), no. 2, 365-370. MR 98k:52010

[8] Delsarte, P., Goethals, J. M. and Seidel, J. J., Spherical codes and designs, Geom. Dedicata, 6 (1977), no. 3, 363-383. MR 58\#5302

[9] Dunford, N. and Schwartz, J., Linear Operators. I. General Theory. Vol. I. Interscience Publishers, New York, 1958. MR 22\#8302

[10] Erdélyi, A., Magnus, W., Oberhettinger, F. and Tricomi, F. G., Higher Transcendental Functions II. McGraw-Hill, New York-Toronto-London, 1953. $M R$ 15,419i

[11] Ewald, G., Larman, D. G. and Rogers, C. A., The directions of the line segments and the $r$-dimensional balls on the boundary of a convex body in Euclidean space, Mathematika, 17 (1970), 1-20. MR 42\#5161

[12] FAlconer, K. J., Applications of a result on spherical integration to the theory of convex sets, Amer. Math. Monthly, 90 (1983), no. 10, 690-693. MR 85f:52012

[13] Funk, P., Über Flächen mit lauter geschlossenen geodätischen Linien, Math. Ann., 74 (1913), no. 2, 278-300. Jahrbuch Fortschr. Math. 44,692

[14] Funk, P., Über eine geometrische Anwendung der Abelschen Integralgleichung, Math. Ann., 77 (1915), no. 1, 129-135. Jahrbuch Fortschr. Math. 45,533

[15] Funk, P., Beiträge zur Theorie der Kugelfunktionen, Math. Ann., 77 (1915), no. 1, 136-152. Jahrbuch Fortschr. Math. 45,702

[16] Gardner, R. J., Geometric Tomography, Encyclopedia of Mathematics and its Applications 58, Cambridge University Press, Cambridge, 1995. MR 96j:52006

[17] Groemer, H., Fourier series and spherical harmonics in convexity, Handbook of Convex Geometry, Vol. A, B, 1259-1295, North-Holland, Amsterdam, 1993. MR 94j:52001 
[18] Groemer, H., Geometric Applications of Fourier Series and Spherical Harmonics, Encyclopedia of Mathematics and its Applications 61, Cambridge University Press, Cambridge, 1996. MR 97j:52001

[19] Hammer, P. C. and Smith, T. J., Conditions equivalent to central symmetry of convex curves, Math. Proc. Cambridge Philos. Soc., 60 (1964), 779-785. MR 30\#506

[20] Hecke, E., Über orthogonal-invariante Integralgleichungen, Math. Ann., 78 (1917), no. 1, 398-404. Jahrbuch Fortschr. Math. 46,632

[21] Heil, E. and Martini, H., Special convex bodies, Handbook of Convex Geometry, Vol. A, B, 347-385, North-Holland, Amsterdam, 1993. MR 94h:52001

[22] Hug, D. and Schneider, R., Stability results involving surface area measures of convex bodies. IV-th Internat. Conf. in "Stochastic Geometry, Convex Bodies, Empirical Measures \& Appls. to Eng. Sci.", Vol. II (Tropea, 2001), Rend. Circ. Mat. Palermo (2), Suppl., 70, part II (2002), 21-51. MR 2004b:52004

[23] Ivanov, B. A., Straight line segments on the boundary of a convex body (Russian), Ukrain. Geom. Sb., No. 13 (1973), 69-71., 2. MR 51\#13864

[24] Lifshitz, I. M. and Pogorelov, A. V., On the determination of Fermi surfaces and electron velocities in metals by the oscillation of magnetic susceptibility (Russian), Dokl. Akad. Nauk SSSR, 96 (1954), 1143-1145. Zbl 57.448

[25] Lassak, M. and Martini, H., Reduced bodies in Minkowski space, Acta Math. Hungar., 106 (2005), no. 1-2, 17-26. MR 2005m:52008

[26] Makai, E., JR., Martini, H. and Ódor, T., Maximal sections and centrally symmetric bodies, Mathematika, 47 (2000), no. 1-2, 19-30. MR 2003e:52005

[27] Makai, E., JR., MARTini, H. and Ódor, T., On an integro-differential transform on the sphere, Studia Sci. Math. Hungar., 38 (2001), 299-312. MR 2002m:44002

[28] Makai, E., JR. and Soltan, V., Lower bounds on the numbers of shadowboundaries and illuminated regions of a convex body, Intuitive Geometry (Szeged, 1991), 249-268, Colloq. Math. Soc. János Bolyai 63, North-Holland, Amsterdam, 1994. MR 97d:52003

[29] Martini, H., Shadow-boundaries of convex bodies, Combinatorics (Acireale, 1992), Discrete Math., 155 (1996), no. 1-3, 161-172. MR 97j:52005

[30] Martini, H. and Swanepoel, K. J., The geometry of Minkowski spaces - a survey. II, Expositiones Math., 22 (2004), no. 2, 93-144. MR 2005h:46028

[31] Martini, H. and Soltan, V., Antipodality properties of finite sets in Euclidean space, Discrete Math., 290 (2005), no. 2-3, 221-228. MR 2005i:52017

[32] Müller, C., Spherical Harmonics, Lecture Notes in Mathematics 17, Springer, Berlin, 1966. MR 33\#7593

[33] Petty, C. M., On Minkowski Geometries, Ph.D. dissertation, University of South California, Los Angeles, 1952.

[34] Petty, C. M., Centroid surfaces, Pacific J. Math., 11 (1961), 1535-1547. MR 24\#A3558

[35] Sansone, G., Orthogonal Functions (Revised ed.), Wiley, Interscience Publishers, London-New York, 1959. MR 21\#2140

[36] Schneider, R., Zu einem Problem von Shephard über die Projektionen konvexer Körper (German), Math. Z., 101 (1967), 71-82. MR 36\#2059

[37] Schneider, R., Functions on a sphere with vanishing integrals over certain subspheres, J. Math. Anal. Appl., 26 (1969), 381-384. MR 38\#6004

[38] SchneIder, R., Über eine Integralgleichung in der Theorie der konvexen Körper, Math. Nachr., 44 (1970), 55-75. MR 43\#1043

[39] Schneider, R., Curvature measures of convex bodies, Ann. Mat. Pura Appl., (4) 116 (1978), 101-134. MR 80d:52012 
[40] Schneider, R., Convex Bodies: The Brunn-Minkowski Theory. Encyclopedia of Mathematics and its Applications 44, Cambridge University Press, Cambridge, 1993. MR 94d:52007

[41] Seeley, R. T., Spherical harmonics, Amer. Math. Monthly 73 (4), Part II, (1966), 115-121. MR 34\#1577

[42] Süss, W., Über Eibereiche mit Mittelpunkt, Math.-Phys. Semesterber., 1 (1950), 273-287. $M R$ 12,46d

[43] Thompson, A. C., Minkowski Geometry. Encyclopedia of Mathematics and its Applications, 63, Cambridge University Press, Cambridge, 1996. MR 97f:52001

[44] Ungar, P., Freak theorem about functions on a sphere, J. London Math. Soc., 29 (1954), 100-103. MR 15,299b

[45] Vilenkin, N. JA., Special Functions and the Theory of Group Representations, Translated from the Russian by V. N. Singh. Translations of Math. Monographs, Vol. 22, AMS, Providence, R. I., 1968. MR 37\#5429.

[46] Vilenkin, N. Ja. and Klimyk, A. U., Representations of Lie Groups and Special Functions. Vol. 2 (English summary), Class 1 Representations, Special Functions, and Integral Transforms, Translated from the Russian by V. A. Groza and A. A. Groza, Math. and its Appls. (Soviet Series) 74, Kluwer Academic Publishers Group, Dordrecht, 1993. MR 94m:22001.

[47] Zindler, K., Über konvexe Gebilde, II. Teil, Monatsh. Math. Phys., 31 (1921), 2557. Jahrbuch Fortschr. Math. 48.0833.05 